\title{
Development
}

\section{Astrocyte-Derived Thrombospondin Induces Cortical Synaptogenesis in a Sex-Specific Manner}

\author{
Anna Mazur, Ean H. Bills, Kayla M. DeSchepper, James C. Williamson, ${ }^{\circledR B}$ Brandon J. Henderson, and \\ DW. Christopher Risher
}

https://doi.org/10.1523/ENEURO.0014-21.2021

Department of Biomedical Sciences, Joan C. Edwards School of Medicine at Marshall University, Huntington, WV 25701

\begin{abstract}
The regulation of synaptic connectivity in the brain is vital to proper functioning and development of the CNS. Formation of neural networks in the CNS has been shown to be heavily influenced by astrocytes, which secrete factors, including thrombospondin (TSP) family proteins, that promote synaptogenesis. However, whether this process is different between males and females has not been thoroughly investigated. In this study, we found that cortical neurons purified from newborn male rats showed a significantly more robust synaptogenic response compared with female-derived cells when exposed to factors secreted from astrocytes. This difference was driven largely by the neuronal response to TSP2, which increased synapses in male neurons while showing no effect on female neurons. Blockade of endogenous $17 \beta$-estradiol (E2) production with letrozole normalized the TSP response between male and female cells, indicating a level of regulation by estrogen signaling. Our results suggest that male and female neurons show a divergent response to TSP synaptogenic signaling, contributing to sex differences in astrocyte-mediated synaptic connectivity.
\end{abstract}

Key words: astrocytes; cortex; estrogen; sex differences; synaptogenesis; thrombospondin

\section{Significance Statement}

The regulation of synaptic connectivity by astrocytes has been a focus of both the neurodevelopmental and glial biology fields for nearly two decades, but many key findings did not take into account the possibility of sex differences. For the first time, we show that a prominent astrocyte signaling factor, thrombospondin-2 (TSP2), strongly promotes synapse formation in male but not female neurons. Furthermore, this sex difference can be abolished by inhibiting production of $17 \beta$-estradiol (E2), revealing that this astrocyte synaptogenic pathway is regulated by estrogen. Our findings should prompt serious consideration into the ramifications of sex and sex hormones on astrocyte synaptic signaling as well as encourage and inform future studies, including potential implications for neurodevelopmental disorders (NDDs) that present with strong sex biases.

\section{Introduction}

Neurons form complex arrangements throughout the CNS that form the basis of our ability to think, move, learn, and remember. Synapses, the basic functional units of the CNS, represent the coming together of an axon from a presynaptic cell and a dendrite from a postsynaptic one. Precise timing and location of synapse formation (i.e., synaptogenesis) is critical for the function of

Received January 12, 2021; accepted July 3, 2021; First published July 15, 2021.

The authors declare no competing financial interests. the developing brain, and it has become increasingly apparent that astrocytes are powerful regulators of this process (Barres, 2008). The last $20+$ years have seen an explosion of discovery of astrocytic mechanisms that influence synaptogenesis, both positively and negatively, via both contact-mediated signaling as well as secreted factors (Allen and Eroglu, 2017). However, despite this greatly increased awareness, a significant caveat of most

Author contributions: W.C.R. designed research; A.M., E.H.B., K.M.D., J.C.W., B.J.H., and W.C.R. performed research; A.M., B.J.H., and W.C.R. analyzed data; A.M. and W.C.R. wrote the paper. 
of these studies was the lack of consideration for potential sex differences in astrocyte-mediated synaptic development.

The brains of males and females show significant structural, chemical, and functional differences (Pfaff and Joëls, 2017). This phenomenon holds true over the course of "normal" development as well as in cases of aberrant CNS function, such as that seen in neurodevelopmental and neurodegenerative disorders (Hanamsagar and Bilbo, 2016). The release of steroids from the gonads beginning with puberty certainly plays a major role in defining sex differences in the still-maturing brain (Genazzani et al., 2007; Ho et al., 2020), but relatively little is known about how prepubertal cellular processes may influence neural circuits in a distinct, sex-dependent manner. Many previous investigations into astrocyte-induced synaptogenesis were performed on neurons isolated from mixed-sex litters of rats and mice, often using astrocyte-conditioned media (ACM) or astrocyte culture inserts which were similarly generated from mixed-sex sources (Allen et al., 2012; Christopherson et al., 2005; Eroglu et al., 2009; Hughes et al., 2010; Kucukdereli et al., 2011; Farhy-Tselnicker et al., 2017; Blanco-Suarez et al., 2018).

As a novel approach, for this study, we used cells isolated specifically from either males or females to identify sex differences in astrocyte-mediated synapse formation. Neuronal cultures isolated from the cortices of male rats experienced a significantly greater fold increase in excitatory synapses when exposed to astrocyte-secreted factors than did cultures purified from females. Driving this sex difference was the strongly synaptogenic response of male-derived neurons to the matricellular protein thrombospondin-2 (TSP2), which was not observed in female cultures. Intriguingly, our data suggest that the divergence may be, at least in part, because of transient differences in de novo neuronal estrogen synthesis during the peak synaptogenic window for astrocytes and TSP.

\section{Materials and Methods}

\section{Animals}

All animal procedures were performed in accordance with the Marshall University Institutional Animal Care and Use Committee's regulations. Adult Sprague Dawley rat mothers and their mixed sex newborn litters were

This work was supported by the National Institutes of Health Grant 1R15MH126345-01 (to W.C.R.), Marshall University startup funds (B.J.H. and W.C.R.), the Marshall University Undergraduate Creative Discovery and Research Scholar Award (E.H.B.), the Marshall University Genomics and Bioinformatics Core, the West Virginia IDeA Network of Biomedical Research Excellence (WV-INBRE) Grant P20GM103434, the COBRE ACCORD Grant 1P20GM121299, and the West Virginia Clinical and Translational Science Institute (WV-CTSI) Grant 2U54GM104942.

Acknowledgements: We thank Dr. Kelly Hopper and the staff of the Marshall University Animal Resource Facility for excellent veterinary assistance.

Correspondence should be addressed to W. Christopher Risher at risherw@ marshall.edu.

https://doi.org/10.1523/ENEURO.0014-21.2021

Copyright @ 2021 Mazur et al.

This is an open-access article distributed under the terms of the Creative Commons Attribution 4.0 International license, which permits unrestricted use, distribution and reproduction in any medium provided that the original work is properly attributed. obtained from Hilltop Lab Animals. Following sex determination (based on visual examination of the anogenital region), pups were separated into male and female before dissection and cell purification.

\section{Cortical neuron purification and treatment}

Cortical neurons were purified separately from male and female postnatal day (P)1 Sprague Dawley rats by sequential immunopanning. Briefly, following rapid decapitation, brains were removed and cortices were dissected. Following $45 \mathrm{~min}$ of digestion in papain $(\sim 7.5$ units $/ \mathrm{ml}$; Worthington) supplemented with DNase (Worthington) dissolved in Dulbecco's PBS (DPBS; Invitrogen) at $34^{\circ}$ $\mathrm{C}$, sequential low/high concentrations of ovomucoid inhibitor (Worthington) was used to gradually halt papain activity. The cell solution was then passaged through a 20- $\mu \mathrm{m}$ Nitex mesh filter (Sefar) before a series of negative immunopanning steps. Petri dishes coated with Bandeiraea Simplicifolia Lectin I (Vector Laboratories), followed by AffiniPure goat-anti mouse $\lg \mathrm{G}+\lg \mathrm{M}(\mathrm{H}+\mathrm{L}$; Jackson ImmunoResearch) and AffiniPure goat-anti rat $\lg \mathrm{G}+\lg \mathrm{M}(\mathrm{H}+\mathrm{L}$; Jackson ImmunoResearch) antibodies were used to remove non-neuronal cells and general debris. To further purify neurons ( $>95 \%$ ) using positive immunopanning, the cell solution was passaged onto Petri dishes coated with mouse antibody against neural cell adhesion molecule L1 (ASCS4; Developmental Studies Hybridoma Bank, University of lowa). Following final washes, pelleting and resuspension in serum-free NGM [Neurobasal (Invitrogen), B27 supplement (Invitrogen), 2 mM GlutaMax (Invitrogen), $100 \mathrm{U} / \mathrm{ml}$ pen/strep (Invitrogen), $1 \mathrm{~mm}$ NaPyruvate (Invitrogen), $50 \mathrm{ng} / \mathrm{ml}$ BDNF (Peprotech), $20 \mathrm{ng} / \mathrm{ml}$ CNTF (Peprotech), $4.2 \mu \mathrm{g} / \mathrm{ml}$ forskolin (Sigma), and $10 \mu \mathrm{M}$ AraC (Sigma)], neurons were plated at a density of $60 \mathrm{~K} /$ well on poly-D-lysine (PDL; Sigma) and laminin (R\&D Systems)-coated coverslips in a 24-well plate. After $2 \mathrm{~d}$ in vitro (DIV2) at $37^{\circ} \mathrm{C} / 5 \% \mathrm{CO}_{2}$, half of the $\mathrm{NGM}$ in each well was replaced with fresh NGM of the same composition with the exception of: Neurobasal Plus (Invitrogen) instead of Neurobasal and GlutaMax, B27 Plus (Invitrogen) instead of $\mathrm{B} 27$, and no AraC; this media was then used for feedings every 2-3d for the duration of the experiment before electrophysiology or fixation for synaptic immunocytochemistry (ICC) on DIV13. Recombinant TSP2 protein was purified from $\mathrm{CHO}$ cells expressing mouse TSP2 via affinity chromatography with HiTRAP heparin HP (GE Healthcare). Standard TSP2 treatment of neurons was performed on DIV7 and DIV10 at a dose of $500 \mathrm{ng} / \mathrm{ml}$.

\section{Cortical astrocyte isolation, culture, and ACM harvesting}

Cortical astrocytes were purified separately from male and female P1 Sprague Dawley rats following a similar protocol to neurons, as described above. After the Nitex mesh filtering step, the cells were pelleted and resuspended in astrocyte growth media [AGM; DMEM + GlutaMax (Invitrogen), 10\% heat-inactivated FBS (Sigma), $10 \mu \mathrm{m}$ hydrocortisone (Sigma), $100 \mathrm{U} / \mathrm{ml}$ pen/strep, $5 \mu \mathrm{g} /$ $\mathrm{ml}$ insulin (Sigma), $1 \mathrm{~mm}$ NaPyruvate, $5 \mu \mathrm{g} / \mathrm{ml} \mathrm{N}$-acetyl-L- 
cysteine (NAC; Sigma)]. After counting, 15-20 million cells were plated on PDL-coated $75-\mathrm{mm}^{2}$ flasks and incubated at $37^{\circ} \mathrm{C} / 5 \% \mathrm{CO}_{2}$. On DIV3, AGM was removed and replaced with DPBS. In order to isolate the adherent monolayer of astrocytes, flasks were shaken vigorously by hand $3-6 \times$ for $15 \mathrm{~s}$ each. DPBS was then replaced with fresh AGM. AraC was added to the AGM from DIV5-DIV7 to minimize astrocyte proliferation. On DIV7, astrocytes were passaged into either transwell inserts (Corning) at a density of $125 \mathrm{~K}$ (for direct culture with neurons) or at 3 million per 100-mm tissue culture dish (for the generation of $\mathrm{ACM}$ ).

For the generation and collection of ACM, at $24 \mathrm{~h}$ postpassaging into $100-\mathrm{mm}$ dishes, astrocytes were washed $3 \times$ with DPBS and conditioned at $37^{\circ} \mathrm{C} / 5 \% \mathrm{CO}_{2}$ without disturbing for $4 \mathrm{~d}$ in minimal medium [Neurobasal (phenol red-free), 2 mm L-g(Invitrogen), 1 mм NaPyruvate, $100 \mathrm{U} /$ $\mathrm{ml}$ pen/strep, $5 \mu \mathrm{g} / \mathrm{ml} \mathrm{NAC,} 40 \mathrm{ng} / \mathrm{ml}$ T3 (Sigma), and Sato supplement (Winzeler and Wang, 2013)]. After conditioning, media was collected from dishes and centrifuged to pellet cell debris before concentrating in 5000 M.W.C.O. Vivaspin tubes (Sartorius). ACM was then aliquoted and flash frozen in liquid nitrogen until use. Standard ACM treatment of neurons was performed on DIV7 and DIV10 at a dose of $75 \mu \mathrm{g} / \mathrm{ml}$ before fixation for synaptic ICC on DIV13.

\section{Synaptic ICC}

DIV13 neurons in 24-well plates were fixed with warm 4\% paraformaldehyde (PFA; Electron Microscopy Sciences) in PBS for 7 min. Next, PFA was removed and cells were washed $3 \times$ with PBS then blocked in a buffer containing $0.2 \%$ Triton X-100 (Roche) in 50\% normal goat serum (NGS; Jackson ImmunoResearch) $/ 50 \%$ antibody buffer (PBS containing $1 \% \mathrm{BSA}, 0.04 \% \mathrm{NaN3}, 0.2 \%$ Triton $\mathrm{X}-100$ ) at room temperature (RT) for $30 \mathrm{~min}$. Following another $3 \times$ PBS wash, cells were treated with $10 \%$ NGS/ $90 \%$ antibody buffer containing primary antibodies against Bassoon (1:500; mouse; Enzo/Assay Designs) and Homer1 (1:500; rabbit; Synaptic Systems), at $4^{\circ} \mathrm{C}$ overnight in the dark. The next morning, another $3 \times$ PBS wash was performed, followed by a 2-h RT incubation in 10\% NGS/90\% antibody buffer containing the following fluorescently-conjugated secondary antibodies: goat anti-mouse Alexa Fluor 488 (1:500; Invitrogen) and goat anti-rabbit Alexa Fluor 594 (1:500; Invitrogen). After final round of $3 \times$ PBS washes, coverslips were transferred to glass slides with Vectashield mounting medium containing DAPI (Nector Laboratories), sealed with nail polish, and imaged on a Leica DM5500B microscope with a $63 \times / 1.4 \mathrm{NA}$ objective at $1920 \times 1440$ resolution.

\section{Whole-cell patch clamp electrophysiology}

Glass coverslips with neuronal cultures were detached from imaging dishes and placed in the recording chamber of an upright microscope (Axio Examiner, Zeiss) while being continually perfused by extracellular solution (ECS; $140 \mathrm{~mm} \mathrm{NaCl}, 5 \mathrm{~mm} \mathrm{KCl}, 2 \mathrm{~mm} \mathrm{CaCl} 2,1 \mathrm{~mm} \mathrm{MgCl} 2,10 \mathrm{~mm}$ HEPES, and $10 \mathrm{~mm}$ glucose). Electrophysiological signals were recorded from visually identified neurons with a
Sutter IPA and SutterPatch software. Patch pipettes were filled with solution containing the following: $135 \mathrm{~mm} \mathrm{~K} \mathrm{glu-}$ conate, $5 \mathrm{~mm} \mathrm{KCl}, 5 \mathrm{~mm}$ EGTA, $0.5 \mathrm{~mm} \mathrm{CaCl}_{2}, 10 \mathrm{~mm}$ HEPES, 2 mm Mg-ATP, and mм 0.1 GTP (pH was adjusted to 7.2 with Tris-base, and osmolarity was adjusted to 280-300 mOsm with sucrose). The resistance of patch pipettes was 4-8 $M \Omega$. Junction potential was nulled just before forming a gigaseal. Series resistance was monitored without compensation throughout the experiment. Data were discarded if the series resistance (10-25 M $\Omega$ ) changed by $>20 \%$ during recordings. All recordings were done at RT. After achieving a gigaseal, gentle suction was used to achieve whole cell configuration. The neuron was voltage clamped at $-70 \mathrm{mV}$ to record miniature EPSCs (mEPSCs) in the presence of $1.0 \mu \mathrm{m}$ tetrodotoxin. Data were sampled at $10 \mathrm{kHz}$ and filtered at $2 \mathrm{kHz}$.

\section{Western blotting}

Western blotting was performed on media collected from DIV10 cultured astrocytes or from cortical neuron lysates prepared on DIV13. For lysate preparation, NGM was aspirated and cells washed twice with TBS $\left(+\mathrm{Mg}^{2+}\right.$ $\left.+\mathrm{Ca}^{2+}\right)$ and lysed in ice-cold solubilization buffer $(25 \mathrm{~mm}$ Tris, $\mathrm{pH} 7.4,150 \mathrm{~mm} \mathrm{NaCl}, 1 \mathrm{~mm} \mathrm{CaCl}_{2}$, and $1 \mathrm{~mm} \mathrm{MgCl}_{2}$ ) containing $0.5 \%$ NP-40 (Thermo Fisher Scientific) and EDTA-free protease inhibitors (Roche). Cells were spun at max speed at $4^{\circ} \mathrm{C}$ for $10 \mathrm{~min}$ and supernatant was collected. The protein concentrations of the lysates were determined using micro-BCA protein assay kit (Pierce); $20-\mu \mathrm{g}$ protein was loaded into each well. Samples were resolved by SDS-PAGE on $4-15 \%$ stain-free polyacrylamide gels (Bio-Rad) and transferred onto a methanol-activated Immobilon-FL PVDF membrane (EMD Millipore). Before blocking, blot was imaged for total protein content on Bio-Rad Chemidoc MP for normalization purposes. Blots were blocked in $50 \%$ fluorescent blocking buffer in PBS (MB-070; Rockland) for $1 \mathrm{~h}$ at RT. Blots were incubated with primary antibody dilution in blocking buffer plus $0.1 \%$ Tween 20: goat anti-TSP2 (AF1635; R\&D Systems); mouse anti-DHP receptor, $\alpha 2$ subunit, 1:500 (D219; Sigma-Aldrich) overnight at $4^{\circ} \mathrm{C}$. Fluorescently labeled secondary antibodies (Multifluor Red and Green, ProteinSimple) were diluted $(1: 1000)$ in the same buffer as primary antibodies, and Western blottings were incubated with secondary antibodies for $1 \mathrm{~h}$ at RT in the dark. Detection was performed using the Bio-Rad Chemidoc MP. Quantification of band intensity was performed with Image Lab software (Bio-Rad) using the imaged total protein blot for normalization.

\section{ELISA for estradiol}

Cortical neurons isolated separately from male and female P1 Sprague Dawley rats were plated at $60 \mathrm{~K} /$ well into 48-well plates coated with PDL and laminin as described previously. Astrocytes were isolated from the same litter as described above, then plated at $600 \mathrm{~K} /$ well into six-well plates on DIV7. Starting on DIV1 (neurons) or DIV5 (astrocytes), medium was collected daily, two wells pooled and flash-frozen per time point, until DIV12. Concentration of estradiol in collected medium was then determined with 
Parameter Estradiol assay (R\&D Systems) according to instructions provided by the manufacturer. Briefly, medium was thawed on ice, centrifuged to precipitate cellular debris, and incubated in ELISA plate wells coated with primary antibody against estradiol, in the presence of fixed amount of HRP-conjugated estradiol. Next, the absorbance was read at $450 \mathrm{~nm}$ and corrected by reading at $570 \mathrm{~nm}$. Data were analyzed using the four-parameter logistic (4-PL) curve-fit, per manufacturer recommendations, generated with Prism software (GraphPad).

\section{Image analysis and statistics}

Synapse quantification was performed using the Puncta Analyzer plugin (Ippolito and Eroglu, 2010) developed by B. Wark for ImageJ (NIH). Analysis was performed by a trained user who was blinded as to condition/ treatment. The plugin allows for rapid counting of presynaptic (i.e., Bassoon), postsynaptic (i.e., Homer1), and colocalized synaptic puncta, determined by user-defined thresholds for each individual channel. This approach provides an accurate estimation of synapse number based on the precise localization of presynaptic and postsynaptic proteins, which are typically non-overlapping when imaged with fluorescence-based ICC except when directly opposed at synapses.

Briefly, the user begins by designating a circular region of interest (ROI) approximately one-cell diameter radially surrounding the neuronal soma. The plugin then separates raw images into "green" (488/Bassoon) and "red" (594/Homer1) channels, subtracts background (rolling ball radius $=50$ ), and then prompts the analyst to define a threshold with the goal of isolating "true" puncta while keeping noise to a minimum (which is facilitated by setting a minimum pixel size value of 4 in the user-selectable settings). The plugin then displays a results window with numbers of presynaptic, postsynaptic, and co-localized synaptic puncta that can be copied to a Microsoft Excel spreadsheet.

Statistical analyses were performed in Microsoft Excel and GraphPad Prism and shown as mean \pm SEM unless otherwise stated, with results summarized in Table 1. In order to calculate "\% of NGM" values, co-localized puncta values (Fig. 1) or frequency/amplitude measurements (Fig. 2) for the same sex GM-only condition were averaged within an experimental replicate, then values across all other treatments/conditions were converted to a percentage of the calculated NGM average for that replicate. For estimation based on confidence intervals (Cls), we entered our raw data values into the appropriate module on https://www.estimationstats.com/ (Ho et al., 2019). Cumming estimation plots showing raw values, mean \pm $\mathrm{SD}$, and $95 \% \mathrm{Cls}$ were downloaded directly from the website, along with the results of comparison testing. A total of 5000 bootstrap samples were taken; the $\mathrm{Cl}$ is bias-corrected and accelerated. Effect sizes and Cls are reported as: effect size $[\mathrm{Cl}$ width lower bound; upper bound]. The $p$ value(s) reported are the likelihood(s) of observing the effect size(s), if the null hypothesis of zero difference is true. For each permutation $p$ value, 5000 reshuffles of the control and test labels were performed.

\section{Results}

\section{Astrocytic contributions to cortical synaptogenesis are differentially regulated between sexes}

To elucidate sex differences in astrocyte-mediated synaptogenesis, we isolated cortical neurons and astrocytes with $>95 \%$ purity from P1 Sprague Dawley rat male or female pups. Neurons were cultured for two weeks in complete NGMs, treated with either astrocyte culture inserts (Astro) or ACM. Following immunocytochemical (ICC) staining, presynaptic (Bassoon) and postsynaptic (Homer1) puncta were imaged and their co-localization quantified to determine excitatory synapse number in the various treatment conditions (Fig. $1 A)$. Astrocyte inserts allow for continuous exchange of secreted factors between astrocytes and neurons via shared media. Both male and female-derived cortical neurons showed a significant increase in excitatory synapse density along their processes when cultured with sex-matched astrocyte inserts (Fig. 1A,B). The unpaired mean difference between $M$ NGM and $M$ Astro was $31.0[95.0 \% \mathrm{Cl} 17.4,45.8]$; between $\mathrm{F} \mathrm{NGM}$ and $\mathrm{F}$ Astro, the difference was $23.2[95.0 \% \mathrm{Cl} 9.28,40.3]$. The $p$ values of the two-sided permutation $t$ tests were 0.0 and 0.0028 , respectively. The percent change (compared with NGM only) of the astrocyte-induced synapses in female cultures was not as high as that in male cultures $(221 \pm 20 \%$ compared with $279 \pm 28 \%$, respectively), though this difference was not significant (Fig. 1B'). To determine whether secreted factors from astrocytes differed in their synaptogenic potential depending on the sex of the donors, we treated male and female-derived cortical neurons with ACM from either male (M-ACM) or female (F-ACM) astrocytes. Excitatory synapse density was increased in neurons cultured from both sexes, with male and female cultures responding significantly to sex-matched ACM (Fig. 1C,D). In the male cultures, the unpaired mean difference between NGM and M-ACM was 18.1 [95.0\% Cl 12.3, 24.4], while between NGM and F-ACM it was $23.5[95.0 \% \mathrm{Cl}$ $15.2,33.3]$; the $p$ values of the two-sided permutation $t$ tests were both 0.0 . In the female cultures, these same comparisons resulted in differences of $6.09[95.0 \% \mathrm{Cl}$ $-2.7,14.3]$ and $14.7[95.0 \% \mathrm{Cl} 4.73,25.1]$, respectively (permutation $t$ test values were 0.163 and 0.0046 , respectively). Interestingly, however, the percent change of the increase in synapses was significantly higher in male compared with female neurons for M-ACM $(207 \pm 14 \%$ in male cultures compared with $157 \pm 19 \%$ in female cultures; $p<0.05)$ and less so for F-ACM $(218 \pm 19 \%$ male, $162 \pm 14 \%$ female; $p=0.169$, Kruskal-Wallis test with Dunn's multiple comparisons post hoc analysis; K-W statistic $=77.03$ (Fig. 1D'). These results indicate that cortical neurons respond to astrocyte-secreted synaptogenic factors at different rates depending on sex.

We next wanted to test whether astrocyte-induced synaptic activity was differently impacted by sex. Male and female cortical neurons were patched in whole-cell 
Table 1: Statistical results for Western blottings, estradiol immunoassay, and ICC (fold-change and multiple group comparisons)

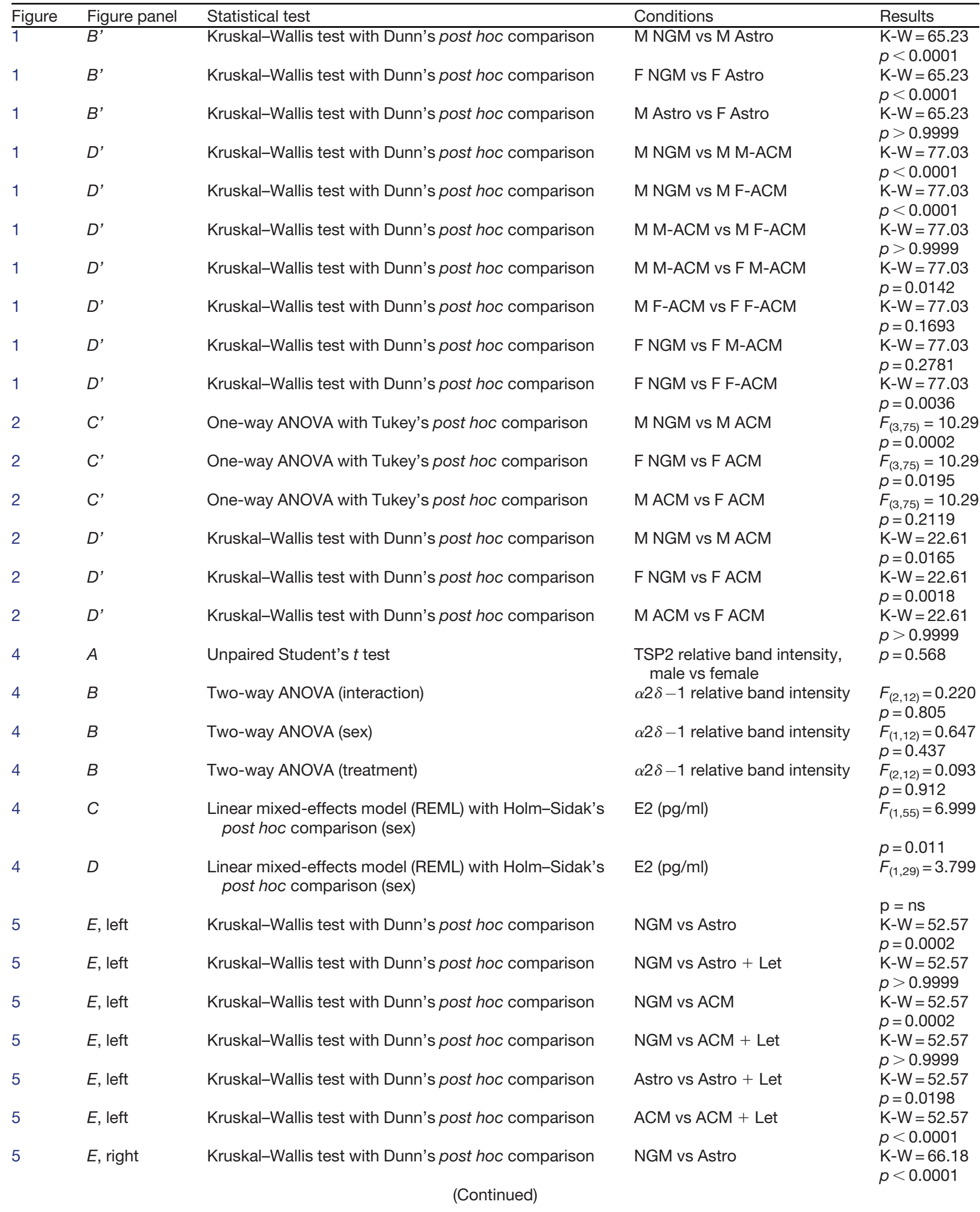


Table 1: Continued

\begin{tabular}{|c|c|c|c|c|}
\hline Figure & Figure panel & Statistical test & Conditions & Results \\
\hline 5 & $E$, right & Kruskal-Wallis test with Dunn's post hoc comparison & NGM vs Astro + Let & $\begin{array}{l}\mathrm{K}-\mathrm{W}=66.18 \\
p=0.0123\end{array}$ \\
\hline 5 & $E$, right & Kruskal-Wallis test with Dunn's post hoc comparison & NGM vs ACM & $\begin{array}{l}\mathrm{K}-\mathrm{W}=66.18 \\
p<0.0001\end{array}$ \\
\hline 5 & $E$, right & Kruskal-Wallis test with Dunn's post hoc comparison & NGM vs ACM + Let & $\begin{array}{l}\mathrm{K}-\mathrm{W}=66.18 \\
p>0.9999\end{array}$ \\
\hline 5 & $E$, right & Kruskal-Wallis test with Dunn's post hoc comparison & Astro vs Astro + Let & $\begin{array}{l}\mathrm{K}-\mathrm{W}=66.18 \\
p=0.2655\end{array}$ \\
\hline 5 & $E$, right & Kruskal-Wallis test with Dunn's post hoc comparison & $A C M$ vs $A C M+$ Let & $\begin{array}{l}\mathrm{K}-\mathrm{W}=66.18 \\
p<0.0001\end{array}$ \\
\hline 6 & left & Kruskal-Wallis test with Dunn's post hoc comparison & NGM vs TSP2 & $\begin{array}{l}\mathrm{K}-\mathrm{W}=64.21 \\
p=0.0039\end{array}$ \\
\hline 6 & left & Kruskal-Wallis test with Dunn's post hoc comparison & NGM vs E2 & $\begin{array}{l}\mathrm{K}-\mathrm{W}=64.21 \\
p=0.2220\end{array}$ \\
\hline 6 & left & Kruskal-Wallis test with Dunn's post hoc comparison & NGM vs E2 TSP2 & $\begin{array}{l}\mathrm{K}-\mathrm{W}=64.21 \\
p>0.9999\end{array}$ \\
\hline 6 & left & Kruskal-Wallis test with Dunn's post hoc comparison & NGM vs ICI & $\begin{array}{l}\mathrm{K}-\mathrm{W}=64.21 \\
p=0.0452\end{array}$ \\
\hline 6 & left & Kruskal-Wallis test with Dunn's post hoc comparison & NGM vs ICI TSP2 & $\begin{array}{l}\mathrm{K}-\mathrm{W}=64.21 \\
p=0.2420\end{array}$ \\
\hline 6 & left & Kruskal-Wallis test with Dunn's post hoc comparison & NGM vs PGE2 & $\begin{array}{l}\mathrm{K}-\mathrm{W}=64.21 \\
p=0.0377\end{array}$ \\
\hline 6 & left & Kruskal-Wallis test with Dunn's post hoc comparison & NGM vs PGE2 TSP2 & $\begin{array}{l}\mathrm{K}-\mathrm{W}=64.21 \\
p=0.0467\end{array}$ \\
\hline 6 & left & Kruskal-Wallis test with Dunn's post hoc comparison & E2 vs E2 TSP2 & $\begin{array}{l}\mathrm{K}-\mathrm{W}=64.21 \\
p>0.9999\end{array}$ \\
\hline 6 & left & Kruskal-Wallis test with Dunn's post hoc comparison & $\mathrm{ICl}$ vs ICI TSP2 & $\begin{array}{l}\mathrm{K}-\mathrm{W}=64.21 \\
p>0.9999\end{array}$ \\
\hline 6 & left & Kruskal-Wallis test with Dunn's post hoc comparison & PGE2 vs PGE2 TSP2 & $\begin{array}{l}K-W=64.21 \\
p>0.9999\end{array}$ \\
\hline 6 & right & Kruskal-Wallis test with Dunn's post hoc comparison & NGM vs TSP2 & $\begin{array}{l}\mathrm{K}-\mathrm{W}=44.75 \\
p>0.9999\end{array}$ \\
\hline 6 & right & Kruskal-Wallis test with Dunn's post hoc comparison & NGM vs E2 & $\begin{array}{l}\mathrm{K}-\mathrm{W}=44.75 \\
p>0.9999\end{array}$ \\
\hline 6 & right & Kruskal-Wallis test with Dunn's post hoc comparison & NGM vs E2 TSP2 & $\begin{array}{l}\mathrm{K}-\mathrm{W}=44.75 \\
p>0.9999\end{array}$ \\
\hline 6 & right & Kruskal-Wallis test with Dunn's post hoc comparison & NGM vs ICI & $\begin{array}{l}\mathrm{K}-\mathrm{W}=44.75 \\
p=0.8729\end{array}$ \\
\hline 6 & right & Kruskal-Wallis test with Dunn's post hoc comparison & NGM vs ICI TSP2 & $\begin{array}{l}\mathrm{K}-\mathrm{W}=44.75 \\
p>0.9999\end{array}$ \\
\hline 6 & right & Kruskal-Wallis test with Dunn's post hoc comparison & NGM vs PGE2 & $\begin{array}{l}K-W=44.75 \\
p>0.9999\end{array}$ \\
\hline 6 & right & Kruskal-Wallis test with Dunn's post hoc comparison & NGM vs PGE2 TSP2 & $\begin{array}{l}\mathrm{K}-\mathrm{W}=44.75 \\
p=0.0002\end{array}$ \\
\hline 6 & right & Kruskal-Wallis test with Dunn's post hoc comparison & E2 vs E2 TSP2 & $\begin{array}{l}\mathrm{K}-\mathrm{W}=44.75 \\
p>0.9999\end{array}$ \\
\hline 6 & right & Kruskal-Wallis test with Dunn's post hoc comparison & $\mathrm{ICl}$ vs ICI TSP2 & $\begin{array}{l}\mathrm{K}-\mathrm{W}=44.75 \\
p=0.6315\end{array}$ \\
\hline 6 & right & Kruskal-Wallis test with Dunn's post hoc comparison & PGE2 vs PGE2 TSP2 & $\begin{array}{l}\mathrm{K}-\mathrm{W}=44.75 \\
p<0.0001\end{array}$ \\
\hline
\end{tabular}

configuration and mEPSCs were recorded to assess baseline synaptic activity (Fig. $2 A, B$ ). Both mEPSC frequency (Fig. 2C) and amplitude (Fig. 2D) increased in response to sex-matched ACM treatment in males and females. Unpaired mean differences between NGM and ACM for frequency comparisons were 0.774 $[95.0 \% \mathrm{Cl} 0.432,1.24]$ for males and $0.783[95.0 \% \mathrm{Cl}$ $0.325,1.36]$ for females, with two-sided permutation $t$ test $p$ values were 0.0008 and 0.0038 , respectively. For amplitude, unpaired mean differences were 5.94 $[95.0 \% \mathrm{Cl} 2.84,9.45]$ for males and $8.98[95.0 \% \mathrm{Cl}$
$3.28,14.9$ ] for females, with $p$ values of 0.0022 and 0.0066 , respectively. Comparison of fold-changes indicates a larger magnitude ACM-induced increase in frequency in male neurons compared with female ( $2.74 \pm 0.46$ compared with $2.01 \pm 0.25$, respectively), though this did not result in a significant difference (Fig. $\left.2 C^{\prime}\right)$. The fold-changes in amplitude increase were similar $\left(1.61 \pm 0.15\right.$ male, $1.46 \pm 0.12$ female; Fig. $\left.2 D^{\prime}\right)$, suggesting that potential sex differences in astrocyte-mediated excitatory synaptogenesis manifest primarily as changes in synapse number rather than strength. 

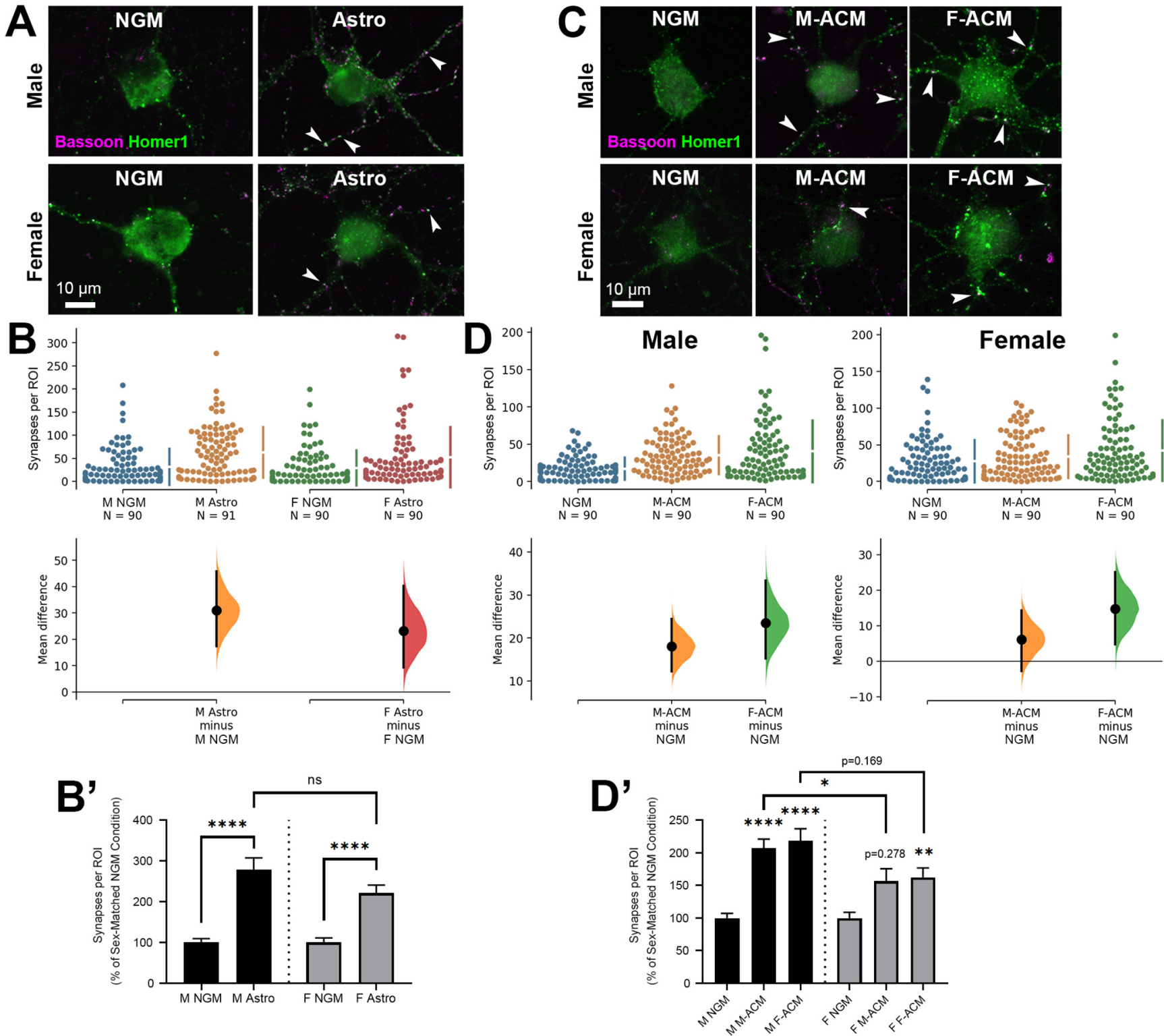

Figure 1. Sex differences in astrocyte-mediated synaptogenesis. $\boldsymbol{A}$, Representative ICC images of cortical neurons isolated from either male (top row) or female (bottom row) pups and treated with either NGM only or cultured with sex-matched astrocyte inserts (Astro) that allow for the continuous exchange of media/factors between cell types. Co-localized presynaptic (Bassoon; magenta) and postsynaptic (Homer1; green) puncta reveal sites of excitatory synapses (white arrowheads). $\boldsymbol{B}$, Exposure to astrocyte-secreted factors via culture inserts significantly increased excitatory synapse number per ROI in both sexes. $\boldsymbol{B}^{\prime}$, Fold-change of synapse number induced by male or female-derived astrocyte inserts (shown as percent of sex-matched NGM control); ${ }^{\star \star \star \star} p<0.0001$; n.s., not significant (Kruskal-Wallis test with Dunn's multiple comparisons post hoc analysis; K-W statistic $=65.23$ ). $\boldsymbol{C}$, Representative ICC images of male (top row) and female (bottom row)-derived cortical neurons treated with ACM previously purified from either males or females (M-ACM and F-ACM, respectively), stained as in $\mathbf{A}$. $\boldsymbol{D}, \mathrm{ACM}$ derived from either sex promoted excitatory synapses in male neurons. In females, this increase was limited to F-ACM. D', Fold-change of synapse number induced by male or female-derived ACM (shown as percent of sex-matched NGM control); ${ }^{\star} p<0.05,{ }^{* *} p<0.01$, ${ }^{\star * \star *} p<0.0001$ (Kruskal-Wallis test with Dunn's multiple comparisons post hoc analysis; K-W statistic $=77.03)$. In $\boldsymbol{B}, \boldsymbol{D}$, the mean difference for each comparison is shown in the Cumming estimation plot. The raw data are plotted on the upper axes; each mean difference (within sex) is plotted on the lower axes as a bootstrap sampling distribution. Mean differences are depicted as dots; $95 \%$ Cls are indicated by the ends of the vertical error bars.

\section{TSP promotes synaptic development in male but not female cortical neurons}

To rule out the possibility that inherent sex differences in synapse density are responsible for the distinct effects of astrocyte-secreted factors on synaptic development, we compared basal synapse number between male and female-derived cortical neuron cultures via ICC (Fig. $3 A$, $B)$. No differences were found in synaptic density between 

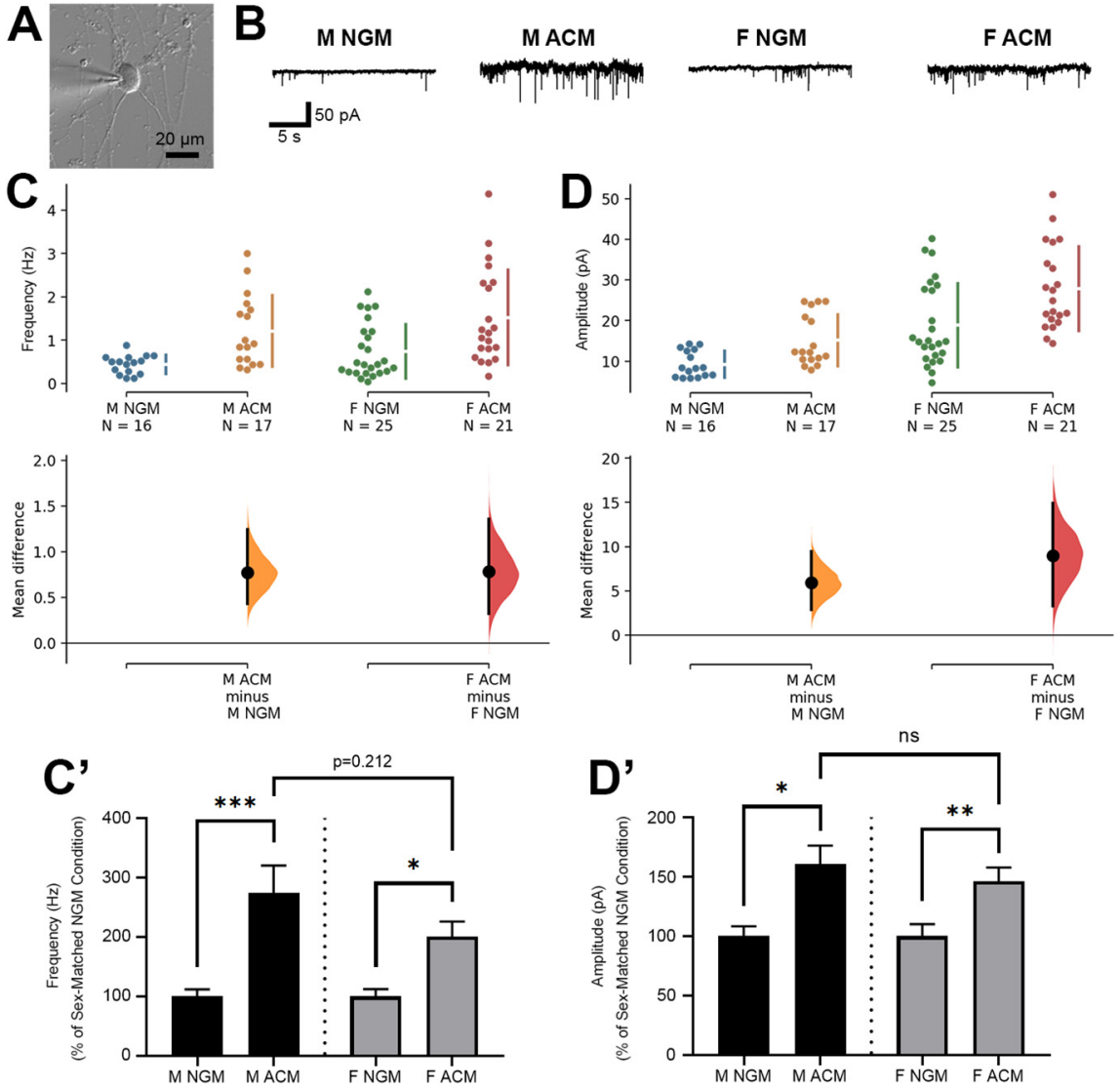

Figure 2. $\boldsymbol{A}$, Brightfield image of a cortical neuron in whole cell patch clamp configuration for the recording of mEPSCs. $\boldsymbol{B}$, Sample mEPSC traces from male and female-derived cortical neurons treated with either standard NGM or NGM with the addition of sexmatched ACM. C and $\boldsymbol{D}$, Sex-matched ACM increased both frequency $(\boldsymbol{C})$ and amplitude $(\boldsymbol{D})$ of mEPSCs recorded from either male or female-derived cortical neurons. C', Fold-change of mEPSC frequency values (in $\mathrm{Hz}$ ) induced by male or female-derived ACM (shown as percent of sex-matched NGM control); ${ }^{*} p<0.05,{ }^{* * *} p<0.001$ (one-way ANOVA with Tukey's multiple comparisons post hoc analysis; $F=10.29$ ). $D^{\prime}$, Fold-change of mEPSC amplitude values (in $\mathrm{pA}$ ) induced by male or female-derived ACM (shown as percent of sex-matched NGM control); ${ }^{*} p<0.05$, ${ }^{* *} p<0.01$; n.s., not significant (Kruskal-Wallis test with Dunn's multiple comparisons post hoc analysis; K-W statistic $=22.61$ ).

the sexes $(0.578[95.0 \% \mathrm{Cl}-2.09,3.35] ; p=0.684)$, indicating that the differences we previously observed must have been because of a differential response to astrocytic factors.

TSPs were among the first astrocyte-secreted factors identified as having synaptogenic properties (Christopherson et al., 2005; Risher and Eroglu, 2012). However, this and other previous studies investigating the synapse-promoting properties of TSP were performed using neurons isolated from mixed-sex litters. To specifically test whether there were sex differences in TSP-induced synaptogenesis, we next analyzed mEPSCs from male versus female cultures treated with purified TSP2 (Fig. $3 C$ ), one of the five synaptogenic TSP isoforms expressed in mammals. Intriguingly, only male-derived neurons showed increased mEPSC frequency after TSP2 treatment (Fig. 3D). The unpaired mean difference between $\mathrm{M}$ GM and M TSP2 was 1.06 [95.0\% $\mathrm{Cl} 0.68,1.77]$; for $\mathrm{F} \mathrm{GM}$ and F TSP2: $-0.0838[95.0 \% \mathrm{Cl}-0.569,0.409] ; p$ values for the two-sided permutation $t$ tests were 0.001 and 0.742 , respectively. Male neurons showed no change in mEPSC amplitude following TSP2 treatment, while female neurons actually showed a slight decrease (M: $-3.27[95.0 \% \mathrm{Cl}$
$-13.4,6.82]$ with $p=0.546 ; F=-5.82[95.0 \% \mathrm{Cl}-10.7$, -2.02] with $p=0.017$; Fig. $3 E$ ), this finding was consistent with previous studies showing that TSPs promote the formation of NMDA-containing silent synapses that lack functional AMPA receptors (Risher and Eroglu, 2012). Nevertheless, these results indicate that TSP2 is synaptogenic in male but not female cortical neurons, providing a potential mechanism underlying the differential rates of astrocyte-induced synaptic development between the sexes.

\section{Modulation of TSP-induced synaptogenesis by estrogen}

Why is TSP effective in promoting synapse formation in male but not female neurons? To begin to address this question, we performed Western blotting to rule out that there were significant sex differences with either astrocytic secretion of TSP2 or expression of the synaptogenic TSP2 receptor, $\alpha 2 \delta-1$ (Eroglu et al., 2009). Media collected from cultured astrocytes showed no difference in secreted TSP2 levels between sexes (Fig. 4A), while $\alpha 2 \delta-1$ was similarly expressed in both male and female- 

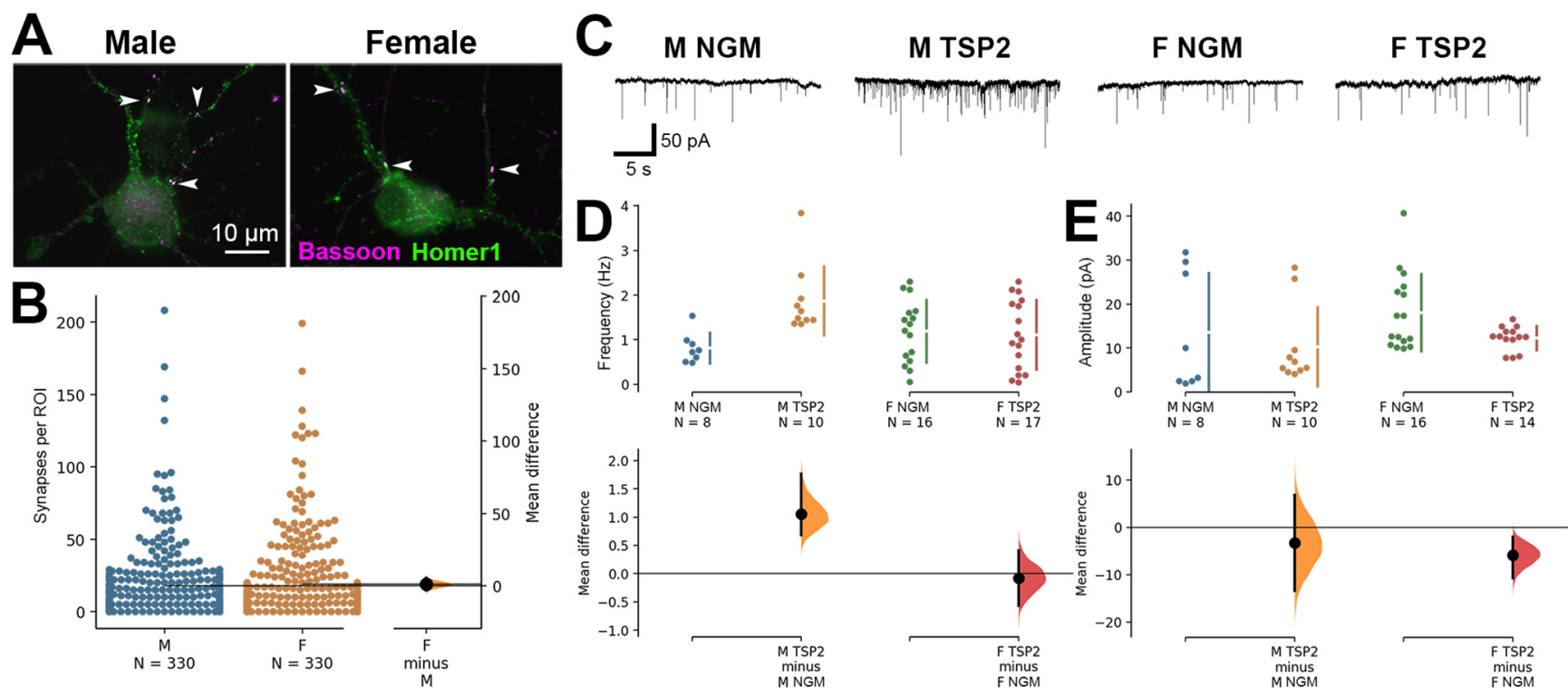

Figure 3. Increased synaptic response to astrocytic factor TSP2 by male but not female-derived cortical neurons. $\boldsymbol{A}$, Representative ICC images of male and female-derived cortical neurons. Co-localized presynaptic (Bassoon; magenta) and postsynaptic (Homer1; green) puncta reveal sites of excitatory synapses (white arrowheads). $\boldsymbol{B}$, No baseline difference in the number of co-localized excitatory synaptic puncta between male and female-derived neurons with standard NGM treatment. The mean difference between M and $\mathrm{F}$ is shown in the Gardner-Altman estimation plot. Both groups are plotted on the left axes; the mean difference is plotted on a floating axis on the right as a bootstrap sampling distribution. The mean difference is depicted as a dot; the $95 \% \mathrm{Cl}$ is indicated by the ends of the vertical error bar. $\boldsymbol{C}$, Sample mEPSC traces from male and female-derived cortical neurons treated with either standard NGM or NGM with the addition of purified TSP2. $\boldsymbol{D}, \boldsymbol{E}$, TSP2 treatment increased frequency $(\boldsymbol{D})$ but not amplitude $(\boldsymbol{E})$ of mEPSCs recorded from male-derived cortical neurons. Female-derived cortical neurons showed no change in mEPSC frequency but had a small decrease in amplitude after TSP2.

derived neuron cultures (Fig. 4B). We then asked whether the unique responses of these cultures were because of differences in the sex hormone estrogen since, like astrocytes, sex hormones have been found to be powerful regulators of synaptic connectivity. Fluctuating levels of circulating estrogen, and androgens to a lesser extent, can drive significant changes in synaptic density in both sexes (Woolley and McEwen, 1992; Leranth et al., 2003; Srivastava and Penzes, 2011). Neurons, including those in dissociated culture, are capable of de novo production and secretion of estrogen (Prange-Kiel et al., 2003; Hojo et al., 2004), which is enzymatically converted from the hormone testosterone by aromatase. We therefore investigated whether application of $17 \beta$-estradiol (E2), the predominant biologically active form of estrogen, or letrozole, an aromatase inhibitor, would affect neuronal $\alpha 2 \delta-1$ protein expression. However, neither E2 nor letrozole had any effect on $\alpha 2 \delta-1$ protein levels in our cultures (Fig. $4 B$ ). We then asked whether there were more subtle differences in endogenous E2 levels that may help explain the TSP2 sex discrepancy. Toward this possibility, Konkle and McCarthy (2011) previously showed that cortical levels of E2 are transiently higher in female rats than males by the end of the first postnatal week. We observed a similar effect in our cortical neuron cultures, reaching significance at DIV9 (Fig. 4C). Interestingly, this time point corresponded with the peak period of synaptogenesis induced by TSPs, as well as the normal developmental expression of TSP1 and TSP2 (Christopherson et al., 2005;
Risher and Eroglu, 2012) and $\alpha 2 \delta-1$ (Risher et al., 2018). By contrast, astrocytes, which have also been identified as sources of estrogen in the brain (Azcoitia et al., 2011), did not show any sex differences in E2 secretion over this same time period (Fig. 4D). These findings suggested that neuronally-derived E2 may be a critical regulator of TSPinduced synaptogenesis.

To investigate whether neuronally-synthesized estrogen interferes with TSP-induced synapse formation, we used ICC to quantify excitatory synapses in our purified cortical neuron cultures (Fig. $5 A, B$ ) following treatment with TSP2 and letrozole. Following nearly two weeks of in vitro culture, ICC strengthened our electrophysiological findings (Fig. 3D) by showing that TSP2 significantly increased synapse numbers in male but not female-derived neurons (Fig. 5C). Intriguingly, treatment with letrozole revealed a sex-divergent effect of estrogen inhibition on the synapse-promoting ability of TSP2. Letrozole facilitated TSP2-induced synaptogenesis in female-derived neurons, albeit not to the same degree as in neurons isolated from males (Fig. $5 \mathrm{C}$ ). The most unexpected result was seen in the male cultures, where letrozole seemed to have the opposite effect, greatly decreasing the magnitude of synaptogenesis induced by TSP2 although it was mildly synaptogenic on its own (Fig. $5 \mathrm{C}$ ). However, the combined effect of TSP2 and letrozole still led to increased synapses over the control treatment in male-derived cells, but now the percent change of this increase was virtually indistinguishable from that seen in the female cultures 


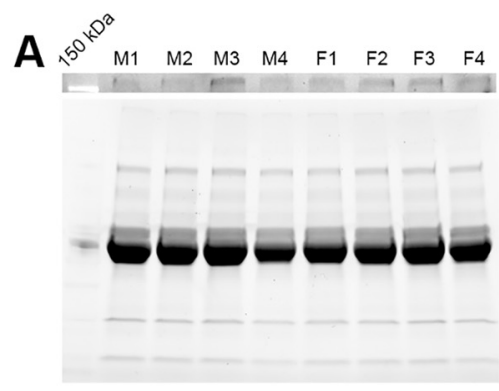

Astrocyte-Secreted TSP2 Protein

B
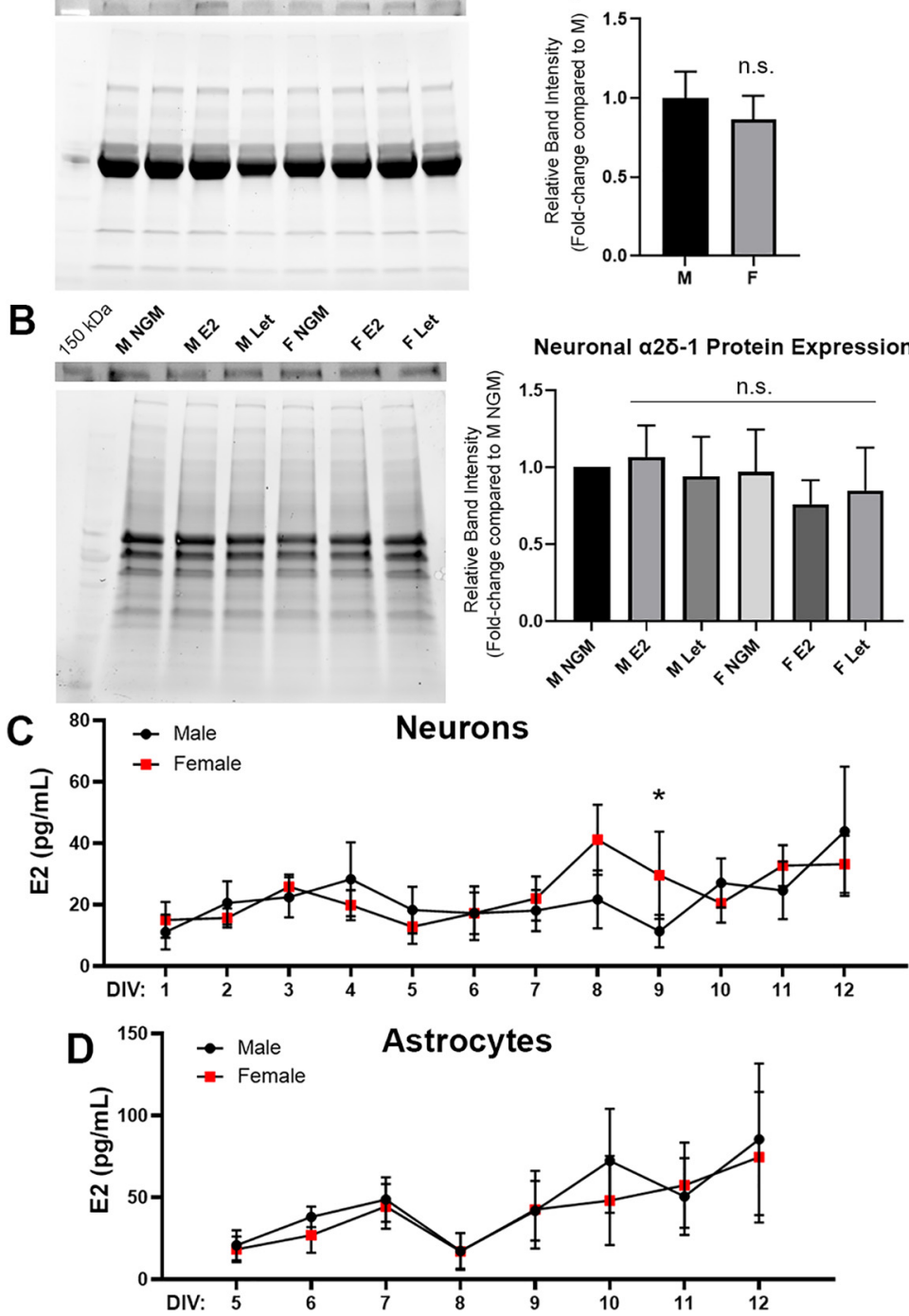

Figure 4. Transient increase in neuronally-derived estradiol in cultured female cortical neurons. $\boldsymbol{A}$, left, Western blotting for TSP2 [top, $\sim 160-\mathrm{kDa}$ observed band ( $\sim 130 \mathrm{kDa}$ expected); bottom, total protein blot for normalization] from astrocyte media collected on DIV10. Right, No difference was found in secreted TSP2 levels between male and female astrocytes; $p=0.568$ [unpaired Student's $t$ test; $n=4$ independent experimental replicates (M1-4, F1-4)]. B, left, Western blotting for $\alpha 2 \delta-1$ (top, $143-k D a$ observed band; bottom, total protein blot for normalization) from purified male and female cortical neuron lysates. Lysates were collected on DIV13 following treatment with NGM only, $100 \mathrm{~nm}$ E2, or $100 \mathrm{~nm}$ letrozole (Let) on DIV7 and DIV10. Right, No difference in total $\alpha 2 \delta-1$ protein expression was observed between male or female-derived neurons at baseline or following treatment with either E2 or Let; $p=0.805$ (two-way ANOVA, interaction: $F_{(2,12)}=0.220 ; n=3$ independent experimental replicates). $\boldsymbol{C}$, Immunoassay results for levels of E2 detected in male versus female neuron-conditioned media. An increase in secreted E2 was detected in female cultures at the start of the second week, reaching significance at DIV9; ${ }^{*} p<0.05$ [linear mixed-effects model (REML) with HolmSidak's post hoc analysis, sex: $F_{(1,55)}=7.00 ; n=4$ independent experimental replicates]. $\boldsymbol{D}$, Estradiol measurements from male versus female cortical ACM. No significant differences were observed (linear mixed-effects model, sex: $F_{(1,29)}=3.80 ; n=3$ independent experimental replicates).

(164 $\pm 15 \%$ male, $160 \pm 21 \%$ female). In male cultures, the unpaired mean difference between NGM and TSP2 was $10.7[95.0 \% \mathrm{Cl} 7.03,14.7]$, between NGM and Let was $5.18[95.0 \% \mathrm{Cl} 2.06,8.83]$, and between NGM and TSP2+Let was $3.13[95.0 \% \mathrm{Cl} 0.685,5.56]$ ( $p$ values for the two-sided permutation $t$ tests were $0.0,0.0012$, and 0.0116 , respectively). In female cultures, the mean differences for those same comparisons were $0.903[95.0 \% \mathrm{Cl}$
$-0.808,2.85],-0.0306$ [95.0\% $\mathrm{Cl}-1.72,1.57]$, and 2.46 $[95.0 \% \mathrm{Cl} 0.436,4.84]$ ( $p$ values of $0.338,0.962$, and 0.0292 , respectively). To test whether the sex difference in the TSP2 response was limited to this specific time period when E2 was transiently elevated in female cultures (Fig. 4C), we performed ICC on neurons with delayed TSP2 and/or letrozole treatment. Compared with our standard treatment timeline (DIV7-DIV13), this delayed 


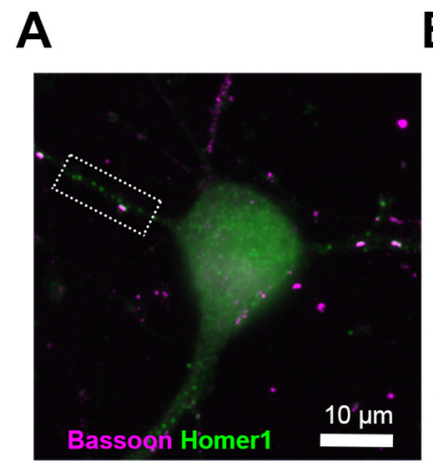

B
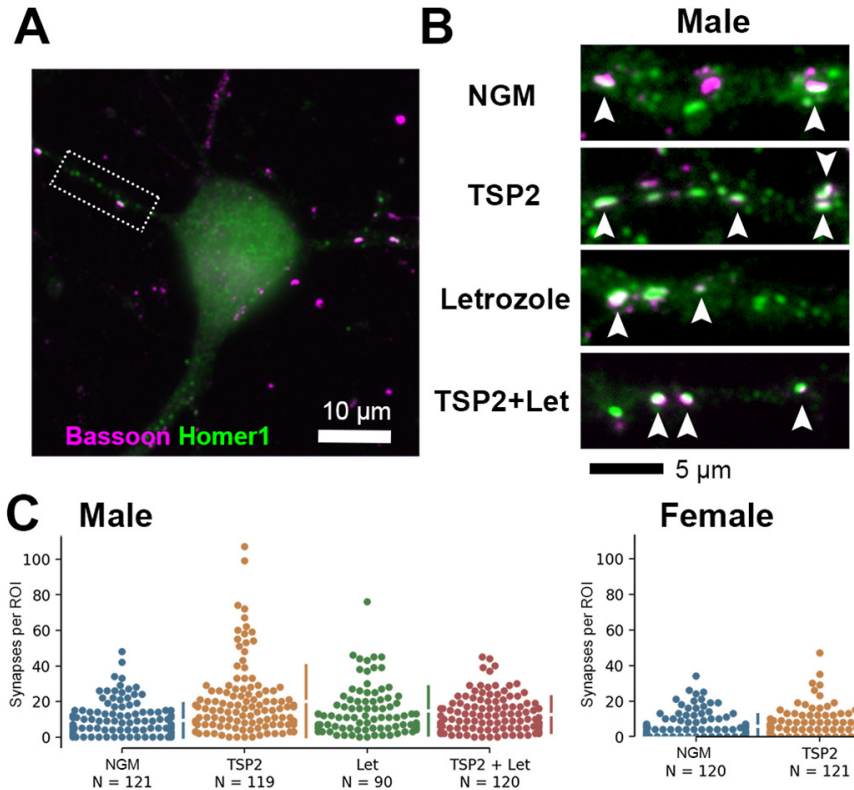

Female
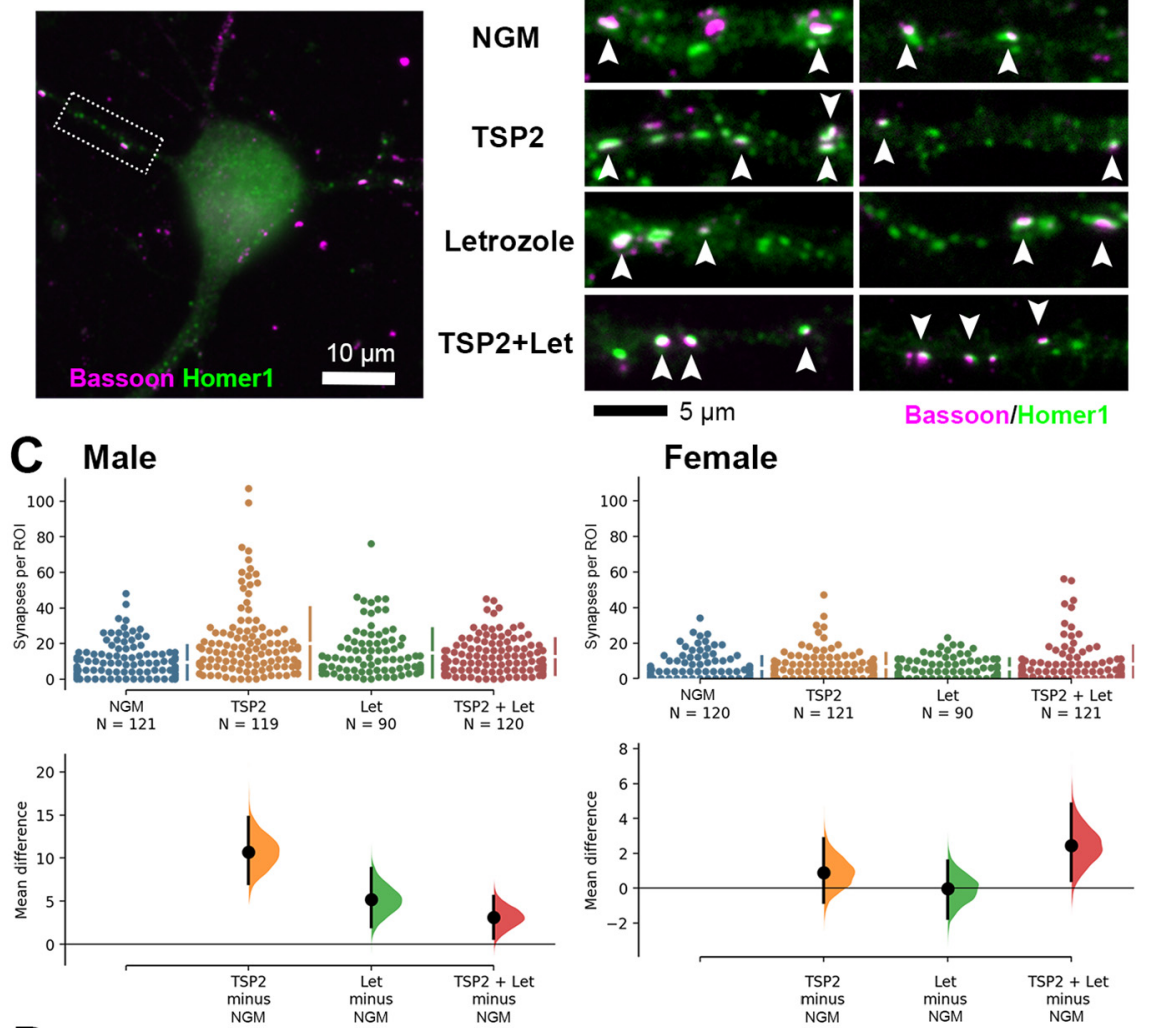

\section{Male (Delayed Tx)}

Female (Delayed Tx)
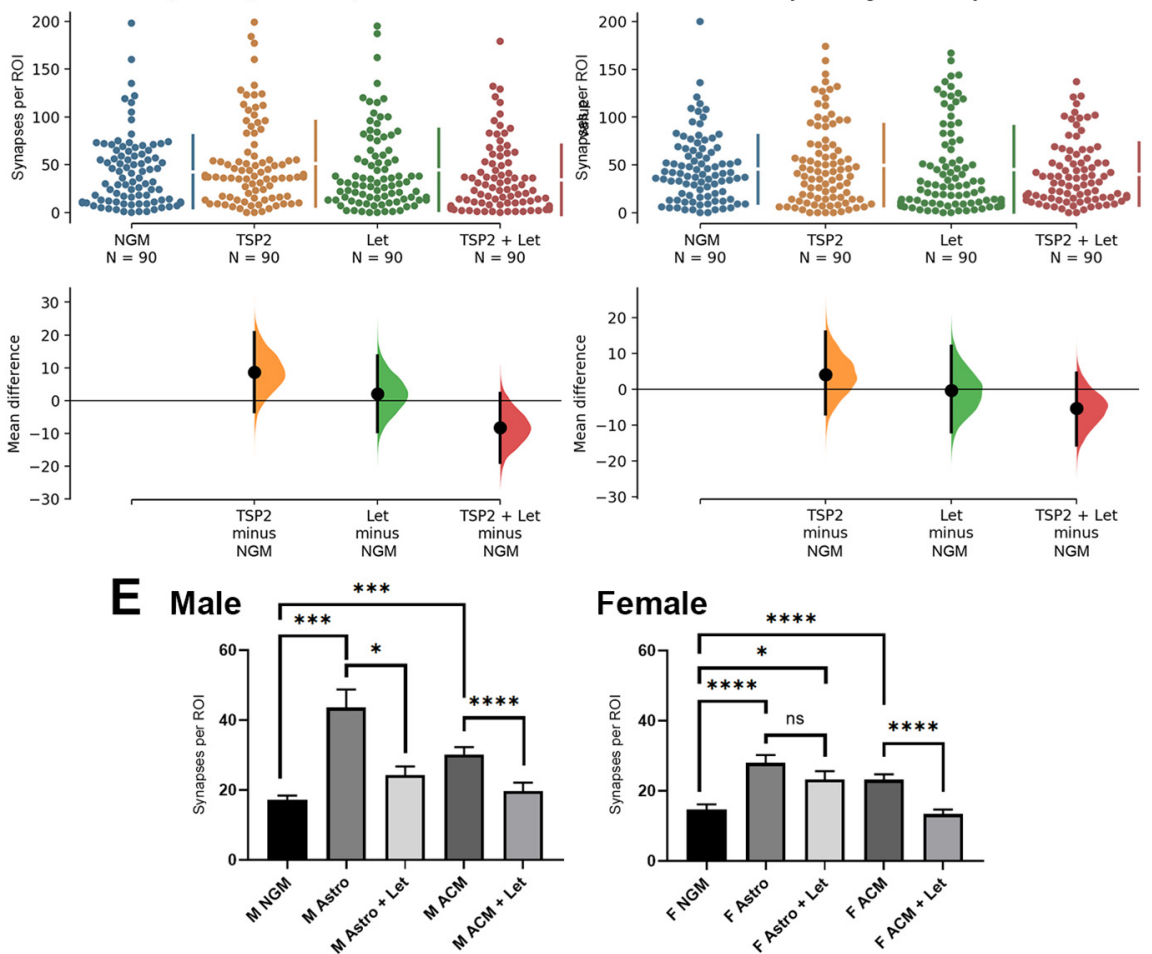

Figure 5. Inhibition of neuronal estrogen production modulates TSP/astrocyte-induced synaptogenesis. A, ICC image of a male-derived cortical neuron fixed and stained on DIV13 with presynaptic (Bassoon; magenta) and postsynaptic (Homer1; green) markers. Dotted box indicates example dendritic ROI sampled for images in $\boldsymbol{B}$, which shows co-localized excitatory synaptic puncta (white, arrowheads) along male and female-derived cortical neurites treated with NGM only or NGM plus TSP2, 100 nM letrozole (Let), or TSP2/letrozole on DIV7 and DIV10. C, left, Letrozole had a mild synaptogenic effect on male-derived cortical neurons but attenuated 
continued

the synapse-promoting ability of TSP2. Right, Female-derived cortical neurons showed no synaptogenic response to TSP2 unless treatment was combined with Let. $\boldsymbol{D}$, Delayed treatment (DIV13 and DIV16, fixed and stained DIV19) revealed no differences in synapse number from NGM-only condition following TSP2 and/or Let treatment in neurons from either sex. $\boldsymbol{E}$, Quantification of Bassoon/Homer1 co-localized synaptic puncta following DIV7/DIV10 treatment schedule showed that Let abolished the synaptogenic effects of sex-matched astrocyte inserts (Astro) and ACM in male-derived neurons as well as ACM in female-derived neurons. No attenuation was observed with Let and Astro in females; ${ }^{\star} p<0.05,{ }^{* \star *} p<0.001,{ }^{* \star \star *} p<0.0001$ [Kruskal-Wallis test with Dunn's multiple comparisons post hoc analysis; K-W statistic $=52.57$ (male), 66.18 (female); $n=30$ cells per condition per experimental replicate, 3 independent experimental replicates].

treatment timeline was performed between DIV13 and DIV19, well after the sex difference in E2 levels had dissipated. We observed no differences from baseline with TSP2 or letrozole in either sex with the delayed treatment (Fig. 5D), supporting the existence of a sex-specific critical window for TSP2-induced synapse formation during the second postnatal week that coincides with the transient difference in E2 levels. For male cultures that received the delayed treatment, the unpaired mean difference between NGM and TSP2 was $8.58[95.0 \% \mathrm{Cl}$ $-3.36,20.7]$, between NGM and Let was $1.99[95.0 \% \mathrm{Cl}$ $-9.49,13.7]$, and between NGM and TSP2+Let was $-8.31[95.0 \% \mathrm{Cl}-18.9,2.28]$ ( $p$ values for the two-sided permutation $t$ tests were $0.167,0.755$, and 0.138 , respectively). In female cultures with delayed treatment, the mean differences for those same comparisons were 4.1 $[95.0 \% \mathrm{Cl}-6.94,16.0],-0.389[95.0 \% \mathrm{Cl}-11.9,12.0]$, and $-5.27[95.0 \% \mathrm{Cl}-15.6,4.49]$ ( $p$ values of 0.485 , 0.947 , and 0.299 , respectively).

Taken together, these results demonstrate clear sex differences in TSP2-induced synaptogenesis that may be regulated, at least in part, by estrogen synthesized endogenously by neurons. To determine whether decreasing estrogen synthesis would specifically interfere with TSP2 signaling pathways or with astrocyte-mediated synaptogenesis in general, we applied letrozole to male and female-derived neuron cultures that were being treated with sex-matched astrocyte inserts or ACM (Fig. 5E). In these treatments, we indeed observed a strong overall attenuation of astrocyte/ACM-induced synapses with letrozole in a similar manner to the reduction seen with TSP2 plus letrozole. Interestingly, letrozole treatment did not reduce the increase in synapses promoted by astrocytes in the female-derived cultures, raising the possibility that inhibiting E2 synthesis may have affected astrocytes in a sexspecific fashion.

We next tested the extent to which TSP2-induced synaptogenesis could be affected by modulating neuronal estrogen signaling beyond the drug letrozole. Using the male and female-derived cortical neuron cultures, we combined TSP2 treatment with one of three other factors involved with estrogen signaling: exogenous E2, ICI 182780 (an estrogen receptor $\alpha / \beta$ antagonist), and prostaglandin E2 (PGE2; aromatase agonist). Whereas inhibiting aromatase with letrozole mildly increased synapse number when combined with TSP2 in both male and female neurons (Fig. 5C), stimulating aromatase activity with PGE2 resulted in a net decrease in synapses with TSP2 treatment (Fig. 6). Furthermore, strongly manipulating estrogen signaling in either direction, either stimu- lating by adding exogenous E2 or inhibiting estrogen receptors with ICI 182780, completely precluded the ability of TSP2 to promote synaptogenesis in either sex (Fig. 6). Combined, these results show that estrogen and TSP2/astrocyte signaling are tightly interlinked in the differential regulation of synaptic connectivity between males and females.

\section{Discussion}

Female and male brains show fundamental differences throughout development and evolution. The adult human female brain estimated to contain approximately twothirds the number of synapses across all cortical layers compared with males (Alonso-Nanclares et al., 2008), raising the possibility of fundamentally sex-distinct mechanisms of how synaptic networks are formed, developed, and maintained. Here, we provide evidence for one such mechanism, whereby cortical neurons purified from males, but not females, are highly receptive to the astrocyte-secreted synaptogenic factor TSP2. Disruption of neuronal estradiol synthesis abolished this sex difference and resulted in TSP2 being weakly synaptogenic for both sexes. In addition, significantly modulating estrogen signaling, positively or negatively (via exogenous E2 or blocking ERs, respectively), completely prevents TSP2induced synaptogenesis, highlighting endogenous brain estrogen as a potentially critical player in astrocyte/synaptic signaling.

TSP is a potent synaptic organizer secreted by astrocytes to act through the neuronal L-type calcium channel auxiliary subunit, $\alpha 2 \delta-1$, during a peak period of synaptogenesis (Eroglu et al., 2009; Risher and Eroglu, 2012). This period consists of roughly the second to third postnatal weeks of rodent brain development, coinciding with a stage of rapid astrocyte growth and elaboration (FarhyTselnicker and Allen, 2018) that is thought to be roughly correlated with perinatal human brain development (Semple et al., 2013). Since this time point falls well before the onset of puberty (typically between days 34 and 48 in rats), neurosteroids have not traditionally been considered to play a significant role in shaping synaptic connectivity. Though we cannot rule out potential contributions from the gonadal testosterone surge near the end of gestation in male rats (Weisz and Ward, 1980), our observation of significant sex differences before the developmental milestone of gonadal hormone release, and the elimination of this difference by letrozole, suggests that our findings are likely because of de novo estrogen synthesis occurring in the very young brain. The phenomenon of de novo estrogen production by brain cells has previously been reported 
(McCarthy, 2008), but the importance of this process for proper neural circuit formation and operation had not been determined.

Our results so far certainly indicate that neuronallysourced estradiol regulates astrocyte-mediated synaptic development, though defining the precise nature of this role has been complicated by the seemingly contradictory finding that blocking aromatase reduces the synaptic capability of TSP2 in male-derived neurons yet is permissive in female cultures. Furthermore, stimulating aromatase via PGE2 appeared to have a negative effect on synaptogenesis in male neurons, while combining PGE2 with TSP2 resulted in decreased synapse number in both sexes (Fig. 6). A number of groups have previously investigated sex differences in aromatase expression throughout the CNS (Bender et al., 2017; Fels et al., 2021; Tabatadze et al., 2014). Though the studies varied in terms of age as well as methodology (mRNA vs protein), a common conclusion is that sex differences do exist in some brain regions but not others. Cortex and hippocampus, for example, are typically cited as areas that do not show significant sex differences in aromatase expression, yet other areas such as the preoptic area of the hypothalamus and the bed nucleus of the stria terminalis (BNST) present with strong sex biases. Since the list of CNS functions influenced by estrogen signaling is quite extensive (McCarthy, 2008), it would stand to reason that blocking aromatase would have widespread consequences that go far beyond the synaptogenic mechanism that we are investigating here. However, there are precedents for sex-specific synaptic responses to letrozole in the hippocampus and basolateral amygdala, despite similar levels of aromatase expression (Bender et al., 2017; Vierk et al., 2012). Further complicating the story is the knowledge that estradiol would not be the sole compound whose synthesis would be affected by letrozole, since aromatase inhibition would make testosterone, the substrate for E2, more readily available for conversion to dihydrotestosterone (DHT) via $5 \alpha$-reductase. DHT acts via androgen receptors (ARs), which have previously been shown to influence synaptic development in a sex-specific manner (Brandt et al., 2020). Studies are ongoing to elucidate this mechanism more fully, as well as to establish the relevance of this process for sex differences in cortical development in vivo.

The role of estrogen as a master regulator of synaptic connectivity has been well-established (for review, see Grkovic and Mitrovic, 2020). However, many previous studies in the field that established the effects of estrogen on synapse formation were performed in conditions that included glia, such as intact brain, hippocampal slice culture, or dissociated neuronal culture with up to $20 \%$ glial contamination (Kretz et al., 2004; Mukai et al., 2007; Kramár et al., 2009; Zhao et al., 2018; Lu et al., 2019; Brandt and Rune, 2020). Both neurons and astrocytes express estrogen receptors (Hösli and Hösli, 1999; Hösli et al., 2001), while astrocytes in particular can rapidly respond to fluctuating levels of estrogen (Chaban et al., 2004), suggesting a potential confound for these findings. In our neuronal cultures, we achieve $95 \%$ purity
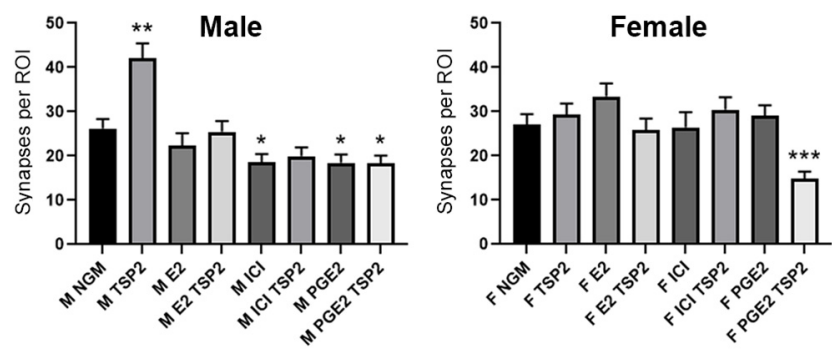

Figure 6. Endogenous estrogen signaling permits TSP2-induced synapse formation in male but not female neurons. Quantification of Bassoon/Homer1 co-localized synaptic puncta following DIV7/DIV10 treatment schedule showed that modulating endogenous estrogen signaling, either with exogenous 100 nм E2, 100 nм ICI 182780 (ER antagonist), or 100 nм PGE2 (aromatase agonist), precluded the ability of TSP2 to promote synapse formation in either male (left)-derived or female (right)derived cortical neurons. PGE2, in particular, was notable in that it lowered synapse number compared with NGM alone in males and when combined with TSP2 in both sexes; ${ }^{*} p<0.05$, ${ }^{\star *} p<0.01,{ }^{* \star *} p<0.001$ [Kruskal-Wallis test with Dunn's multiple comparisons post hoc analysis; K-W statistic $=64.21$ (male), 44.75 (female); $n=30$ cells per condition per experimental replicate, 3 independent experimental replicates].

(confirmed by ICC) and include cytosine arabinoside (AraC) to inhibit the proliferation of the astrocytes that are present. In this way, we are able to isolate the responses of neurons and specifically provide the factors typically sourced from astrocytes, either as individual components (i.e., TSPs) or as a heterogeneous mixture (i.e., ACM). As the findings in this study have indicated, such an approach may be necessary to continue unraveling the intersection between estrogen and astrocytes in the shaping of synaptic connectivity (McCarthy et al., 2003).

In addition to the known differences between male and female brains of healthy individuals, aberrant brain states are also known to manifest differently between the sexes. Diseases of the aging brain, such as Alzheimer's and Parkinson's, present with highly different rates of incidence and severity between men and women, while many more boys than girls are diagnosed with certain neurodevelopmental disorders (NDDs) including schizophrenia and autism spectrum disorder (Abel et al., 2010; Werling and Geschwind, 2013; Hanamsagar and Bilbo, 2016). In the case of NDDs, it is tempting to speculate that early dysregulation of synaptic connectivity may contribute to disease pathogenesis in a sex-dependent manner. Indeed, synaptic pathology and aberrant spinogenesis are common findings in both autism and schizophrenia, with a number of overlapping candidate molecules between the disorders (including TSP receptor $\alpha 2 \delta-1$ and its associated calcium channel subunits) that suggest some degree of common mechanistic dysfunction (Penzes et al., 2011; lossifov et al., 2014; Risher et al., 2018; Harrison et al., 2020). Future studies that aim to elucidate disease pathology for the sake of developing novel, targeted therapies (Gillies and McArthur, 2010) should therefore take into consideration whether astrocyte-mediated synaptic signaling pathways are differentially regulated and/or disturbed in one sex compared with the other. 
In summary, our findings have revealed that at least one prominent synaptogenic pathway regulated by astrocytes displays a significant sex bias, with TSP2 promoting synapse formation in male but not female cortical neurons. Future studies investigating other astrocyte factors, such as TSP1 and TSP4, hevin (Risher et al., 2014), SPARC (Kucukdereli et al., 2011), glypicans (Allen et al., 2012; Farhy-Tselnicker et al., 2017), and TGF- $\beta$ (Diniz et al., 2014), among others, may determine whether this phenomenon affects other glial signaling pathways or is specific to TSP2. From this starting point, we can start to more thoroughly investigate how astrocytes promote synaptic connectivity in both sexes, elucidating potential mechanisms that underlie fundamental differences in the male and female brain to address an understudied yet vitally important area of neuroscience (McCarthy et al., 2012).

\section{References}

Abel KM, Drake R, Goldstein JM (2010) Sex differences in schizophrenia. Int Rev Psychiatry 22:417-428.

Allen NJ, Eroglu C (2017) Cell biology of astrocyte-synapse interactions. Neuron 96:697-708.

Allen NJ, Bennett ML, Foo LC, Wang GX, Chakraborty C, Smith SJ, Barres BA (2012) Astrocyte glypicans 4 and 6 promote formation of excitatory synapses via GluA1 AMPA receptors. Nature 486:410-414.

Alonso-Nanclares L, Gonzalez-Soriano J, Rodriguez JR, DeFelipe J (2008) Gender differences in human cortical synaptic density. Proc Natl Acad Sci USA 105:14615-14619.

Azcoitia I, Yague JG, Garcia-Segura LM (2011) Estradiol synthesis within the human brain. Neuroscience 191:139-147.

Barres BA (2008) The mystery and magic of glia: a perspective on their roles in health and disease. Neuron 60:430-440.

Bender RA, Zhou L, Vierk R, Brandt N, Keller A, Gee CE, Schäfer MK, Rune GM (2017) Sex-dependent regulation of aromatase-mediated synaptic plasticity in the basolateral amygdala. J Neurosci 37:1532-1545.

Blanco-Suarez E, Liu TF, Kopelevich A, Allen NJ (2018) Astrocyte-secreted chordin-like 1 drives synapse maturation and limits plasticity by increasing synaptic GluA2 AMPA receptors. Neuron 100:1116-1132.e3.

Brandt N, Rune GM (2020) Sex-dependency of oestrogen-induced structural synaptic plasticity: inhibition of aromatase versus application of estradiol in rodents. Eur J Neurosci 52:2548-2559.

Brandt N, Vierk R, Fester L, Anstötz M, Zhou L, Heilmann LF, Kind S, Steffen P, Rune GM (2020) Sex-specific difference of hippocampal synaptic plasticity in response to sex neurosteroids. Cereb Cortex 30:2627-2641.

Chaban VV, Lakhter AJ, Micevych P (2004) A membrane estrogen receptor mediates intracellular calcium release in astrocytes. Endocrinology 145:3788-3795.

Christopherson KS, Ullian EM, Stokes CC, Mullowney CE, Hell JW, Agah A, Lawler J, Mosher DF, Bornstein P, Barres BA (2005) Thrombospondins are astrocyte-secreted proteins that promote CNS synaptogenesis. Cell 120:421-433.

Diniz LP, Matias IC, Garcia MN, Gomes FC (2014) Astrocytic control of neural circuit formation: highlights on TGF-beta signaling. Neurochem Int 78:18-27.

Eroglu C, Allen NJ, Susman MW, O'Rourke NA, Park CY, Ozkan E, Chakraborty C, Mulinyawe SB, Annis DS, Huberman AD, Green EM, Lawler J, Dolmetsch R, Garcia KC, Smith SJ, Luo ZD, Rosenthal A, Mosher DF, Barres BA (2009) Gabapentin receptor alpha2delta-1 is a neuronal thrombospondin receptor responsible for excitatory CNS synaptogenesis. Cell 139:380-392.
Farhy-Tselnicker I, Allen NJ (2018) Astrocytes, neurons, synapses: a tripartite view on cortical circuit development. Neural Dev 13:7.

Farhy-Tselnicker I, van Casteren ACM, Lee A, Chang VT, Aricescu AR, Allen NJ (2017) Astrocyte-secreted glypican 4 regulates release of neuronal pentraxin 1 from axons to induce functional synapse formation. Neuron 96:428-445.e13.

Fels JA, Casalena GA, Manfredi G (2021) Sex and oestrogen receptor beta have modest effects on gene expression in the mouse brain posterior cortex. Endocrinol Diabetes Metab 4:e00191.

Genazzani AR, Pluchino N, Luisi S, Luisi M (2007) Estrogen, cognition and female ageing. Hum Reprod Update 13:175-187.

Gillies GE, McArthur S (2010) Estrogen actions in the brain and the basis for differential action in men and women: a case for sex-specific medicines. Pharmacol Rev 62:155-198.

Grkovic I, Mitrovic N (2020) Estradiol induces synaptic rearrangements. Vitam Horm 114:233-256.

Hanamsagar R, Bilbo SD (2016) Sex differences in neurodevelopmental and neurodegenerative disorders: focus on microglial function and neuroinflammation during development. J Steroid Biochem Mol Biol 160:127-133.

Harrison PJ, Tunbridge EM, Dolphin AC, Hall J (2020) Voltage-gated calcium channel blockers for psychiatric disorders: genomic reappraisal. Br J Psychiatry 216:250-253.

Ho J, Tumkaya T, Aryal S, Choi H, Claridge-Chang A (2019) Moving beyond $P$ values: data analysis with estimation graphics. Nat Methods 16:565-566.

Ho TC, Colich NL, Sisk LM, Oskirko K, Jo B, Gotlib IH (2020) Sex differences in the effects of gonadal hormones on white matter microstructure development in adolescence. Dev Cogn Neurosci 42:100773.

Hojo Y, Hattori TA, Enami T, Furukawa A, Suzuki K, Ishii HT, Mukai $\mathrm{H}$, Morrison JH, Janssen WG, Kominami S, Harada N, Kimoto T, Kawato S (2004) Adult male rat hippocampus synthesizes estradiol from pregnenolone by cytochromes P45017alpha and P450 aromatase localized in neurons. Proc Natl Acad Sci USA 101:865870.

Hösli E, Hösli L (1999) Cellular localization of estrogen receptors on neurones in various regions of cultured rat CNS: coexistence with cholinergic and galanin receptors. Int J Dev Neurosci 17:317-330.

Hösli E, Jurasin K, Rühl W, Luthy R, Hösli L (2001) Colocalization of androgen, estrogen and cholinergic receptors on cultured astrocytes of rat central nervous system. Int J Dev Neurosci 19:11-19.

Hughes EG, Elmariah SB, Balice-Gordon RJ (2010) Astrocyte secreted proteins selectively increase hippocampal GABAergic axon length, branching, and synaptogenesis. Mol Cell Neurosci 43:136145.

Iossifov I, O’Roak BJ, Sanders SJ, Ronemus M, Krumm N, Levy D, Stessman HA, Witherspoon KT, Vives L, Patterson KE, Smith JD, Paeper B, Nickerson DA, Dea J, Dong S, Gonzalez LE, Mandell JD, Mane SM, Murtha MT, Sullivan CA, et al. (2014) The contribution of de novo coding mutations to autism spectrum disorder. Nature 515:216-221.

Ippolito DM, Eroglu C (2010) Quantifying synapses: an immunocytochemistry-based assay to quantify synapse number. J Vis Exp. Advance online publication. Retrieved Nov 16, 2010. doi: 10.3791/ 2270.

Konkle AT, McCarthy MM (2011) Developmental time course of estradiol, testosterone, and dihydrotestosterone levels in discrete regions of male and female rat brain. Endocrinology 152:223-235.

Kramár EA, Chen LY, Brandon NJ, Rex CS, Liu F, Gall CM, Lynch G (2009) Cytoskeletal changes underlie estrogen's acute effects on synaptic transmission and plasticity. J Neurosci 29:12982-12993.

Kretz O, Fester L, Wehrenberg U, Zhou L, Brauckmann S, Zhao S, Prange-Kiel J, Naumann T, Jarry H, Frotscher M, Rune GM (2004) Hippocampal synapses depend on hippocampal estrogen synthesis. J Neurosci 24:5913-5921.

Kucukdereli H, Allen NJ, Lee AT, Feng A, Ozlu MI, Conatser LM, Chakraborty C, Workman G, Weaver M, Sage EH, Barres BA, Eroglu C (2011) Control of excitatory CNS synaptogenesis by 
astrocyte-secreted proteins Hevin and SPARC. Proc Natl Acad Sci USA 108:E440-E449.

Leranth C, Petnehazy O, MacLusky NJ (2003) Gonadal hormones affect spine synaptic density in the CA1 hippocampal subfield of male rats. J Neurosci 23:1588-1592.

Lu Y, Sareddy GR, Wang J, Wang R, Li Y, Dong Y, Zhang Q, Liu J, O'Connor JC, Xu J, Vadlamudi RK, Brann DW (2019) Neuron-derived estrogen regulates synaptic plasticity and memory. J Neurosci 39:2792-2809.

McCarthy MM (2008) Estradiol and the developing brain. Physiol Rev 88:91-124.

McCarthy MM, Todd BJ, Amateau SK (2003) Estradiol modulation of astrocytes and the establishment of sex differences in the brain. Ann NY Acad Sci 1007:283-297.

McCarthy MM, Arnold AP, Ball GF, Blaustein JD, De Vries GJ (2012) Sex differences in the brain: the not so inconvenient truth. J Neurosci 32:2241-2247.

Mukai H, Tsurugizawa T, Murakami G, Kominami S, Ishii H, OgiueIkeda M, Takata N, Tanabe N, Furukawa A, Hojo Y, Ooishi Y, Morrison JH, Janssen WG, Rose JA, Chambon P, Kato S, Izumi S, Yamazaki T, Kimoto T, Kawato S (2007) Rapid modulation of longterm depression and spinogenesis via synaptic estrogen receptors in hippocampal principal neurons. J Neurochem 100:950-967.

Penzes P, Cahill ME, Jones KA, VanLeeuwen JE, Woolfrey KM (2011) Dendritic spine pathology in neuropsychiatric disorders. Nat Neurosci 14:285-293.

Pfaff DW, Joëls M (2017) Hormones, brain, and behavior. Amsterdam; Boston: Elsevier/Academic Press.

Prange-Kiel J, Wehrenberg U, Jarry H, Rune GM (2003) Para/autocrine regulation of estrogen receptors in hippocampal neurons. Hippocampus 13:226-234.

Risher WC, Eroglu C (2012) Thrombospondins as key regulators of synaptogenesis in the central nervous system. Matrix Biol 31:170177.

Risher WC, Patel S, Kim IH, Uezu A, Bhagat S, Wilton DK, Pilaz LJ, Singh Alvarado J, Calhan OY, Silver DL, Stevens B, Calakos N,
Soderling SH, Eroglu C (2014) Astrocytes refine cortical connectivity at dendritic spines. Elife 3:e04047.

Risher WC, Kim N, Koh S, Choi JE, Mitev P, Spence EF, Pilaz LJ, Wang D, Feng G, Silver DL, Soderling SH, Yin HH, Eroglu C (2018) Thrombospondin receptor $\alpha 2 \delta-1$ promotes synaptogenesis and spinogenesis via postsynaptic Rac1. J Cell Biol 217:3747-3765.

Semple BD, Blomgren K, Gimlin K, Ferriero DM, Noble-Haeusslein LJ (2013) Brain development in rodents and humans: identifying benchmarks of maturation and vulnerability to injury across species. Prog Neurobiol 106-107:1-16.

Srivastava DP, Penzes P (2011) Rapid estradiol modulation of neuronal connectivity and its implications for disease. Front Endocrinol (Lausanne) 2:77.

Tabatadze N, Sato SM, Woolley CS (2014) Quantitative analysis of long-form aromatase mRNA in the male and female rat brain. PLoS One 9:e100628.

Vierk R, Glassmeier G, Zhou L, Brandt N, Fester L, Dudzinski D, Wilkars W, Bender RA, Lewerenz M, Gloger S, Graser L, Schwarz J, Rune GM (2012) Aromatase inhibition abolishes LTP generation in female but not in male mice. J Neurosci 32:8116-8126.

Weisz J, Ward IL (1980) Plasma testosterone and progesterone titers of pregnant rats, their male and female fetuses, and neonatal offspring. Endocrinology 106:306-316.

Werling DM, Geschwind DH (2013) Sex differences in autism spectrum disorders. Curr Opin Neurol 26:146-153.

Winzeler A, Wang JT (2013) Purification and culture of retinal ganglion cells from rodents. Cold Spring Harb Protoc 2013:643-652.

Woolley CS, McEwen BS (1992) Estradiol mediates fluctuation in hippocampal synapse density during the estrous cycle in the adult rat. J Neurosci 12:2549-2554.

Zhao J, Bian C, Liu M, Zhao Y, Sun T, Xing F, Zhang J (2018) Orchiectomy and letrozole differentially regulate synaptic plasticity and spatial memory in a manner that is mediated by SRC-1 in the hippocampus of male mice. J Steroid Biochem Mol Biol 178:354368. 